\title{
La scienza medica nei papiri ercolanesi
}

\author{
Gianluca Del Mastro
}

Dalla lettura dei papiri carbonizzati di Ercolano, come è stato più volte sottolineato, emerge il ruolo della filosofia epicurea come farmaco dell'anima. In molti casi, gli autori epicurei riprendono la terminologia medica per descrivere i sintomi e le terapie che consentono di combattere i mali che impediscono all'uomo di raggiungere la felicità. Nel corso di questo breve studio, intendo riprendere molti di questi passi e considerarne alcuni nuovi alla luce degli studi più recenti.

A partire dagli studi di Jaeger ${ }^{1}$ sul metodo di Platone, che preferì un modello medico a quello matematico per descrivere gli sviluppi etici del sistema filosofico, diversi studiosi ${ }^{2}$ hanno messo in evidenza la stretta connessione tra la filosofia e la medicina, proprio a partire da Democrito e Platone e il riconoscimento del filosofo, in special modo di quello ellenistico, che, come terapeuta dell'anima, indica il percorso da seguire per il raggiungimento di un determinato fine etico.

Gli studi di Wolfgang Schmid, in particolare la voce "Epikur" nel Reallexikon für Antike und Christentum ${ }^{3}$, costituiscono un punto di partenza imprescindibile per l'identificazione dei frammenti epicurei da cui emerge il ruolo del filosofo come medico e della filosofia come guida dell'anima.

Le Ratae Sententiae I-IV riportate alla fine del X libro delle Vite dei filosofi di

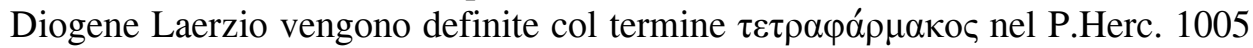
(un'opera polemica di Filodemo Contro coloro che affermano di conoscere le opere dei Maestri $)^{4}$. La critica è stata a lungo discorde se $\tau \varepsilon \tau \rho \alpha \varphi \alpha ́ p \mu \alpha \kappa o \varsigma$ fosse stato adottato da Epicuro o da un suo successore ${ }^{5}$. L'uso del termine nel papiro,

\footnotetext{
${ }^{1}$ Jaeger, Paideia III.

${ }^{2}$ Nella imponente bibliografia sull'argomento, mi limito a rimandare a Voelke, Philosophie, a Hadot, Philosophie, (in part. pp. 161-2, 180, 192, 222, 326, 330, 366-7) e alle importanti riflessioni di Nussbaum, Therapy (in part. i capp. 1-3).

${ }^{3}$ Schmid, Epikur.

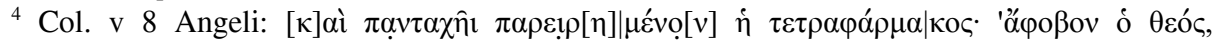

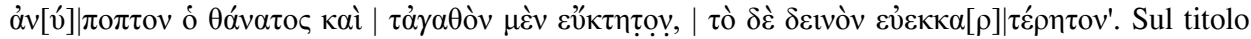
dell'opera, cf. Del Mastro, Titoli, 185-7.

${ }^{5}$ Cf. Angeli, Amici, 50 ss. e 265 ss.
} 
in un contesto in cui vengono riferite le parole del Maestro, sembra inequivocabile: i capisaldi della dottrina (l'allontanamento del timore degli dei e della morte, la facilità di perseguire il bene e di sopportare il dolore) vengono calati in

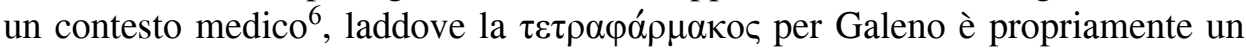
unguento di cera, resina, pece e grasso, impiegato per curare le infiammazioni purulente, le $\varphi \lambda \varepsilon \gamma \mu \mathrm{ov \alpha i} i^{7}$.

Nella Sentenza Vaticana $54^{8}$ Epicuro afferma che "non bisogna fingere di filosofare, ma filosofare davvero; non abbiamo infatti bisogno di apparire sani, ma di esserlo davvero"9. In questo caso, è significativo l'uso del verbo viriaíveıv, impiegato per indicare la salute dell'anima. In un altro caso, nell'Epistola a Meneceo $^{10}$, la $v \gamma t \varepsilon i ́ \alpha$, che indica il buono stato del corpo, insieme all'á $\tau \alpha \rho \alpha \xi^{\prime \prime} \alpha$

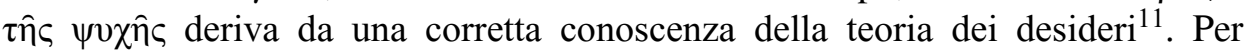
Epicuro le virtù "non sono scelte per se stesse, ma per il piacere, come la medicina per la salute"12. L'uso della terminologia medica è spia dell'importanza terapeutica che Epicuro attribuiva alla filosofia e alla stretta connessione tra le due scienze, che in Lucrezio si traduce nella possibilità di curare l'anima attraverso la terapia medica, così come avviene per il corpo. Questa similitudine costituisce una prova ulteriore della mortalità dell'anima: et quoniam mentem sanari, corpus ut aegrum, / cernimus et flecti medicina posse videmus / id quoque praesagit mortalem vivere mentem ${ }^{13}$.

Marcello Gigante, in un importante articolo pubblicato nelle "Cronache Ercolanesi” del $1975^{14}$, dal titolo paradigmatico Philosophia medicans in Filodemo,

\footnotetext{
${ }^{6} \mathrm{Cf}$. Angeli, Compendi, 59-66.

${ }^{7}$ Elem. ex Hipp. libri II, I $452 \mathrm{~K}$.

${ }^{8}$ Fr. 220 Usener.

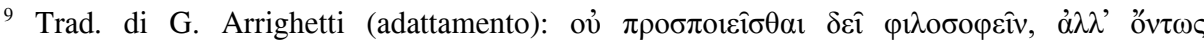

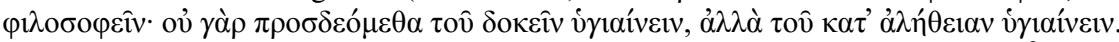

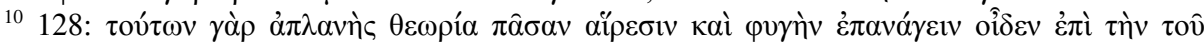

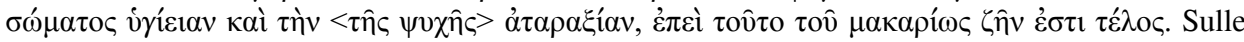
difficoltà della tradizione testuale rimando all'apparato critico di Dorandi, Diogenes, 808 e a Hessler, Epikur, 241-2.

${ }^{11}$ Nel P.Herc. 1251 (un'opera attribuita a Filodemo, Sulle scelte e $i$ rifiuti), a col. ii 7-10 Indelli-Tsouna McKirahan, sembra che Filodemo attacchi dei Cirenaici o degli Scettici i quali

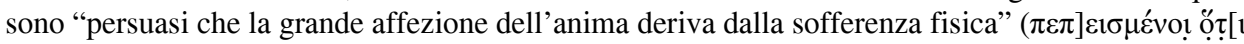

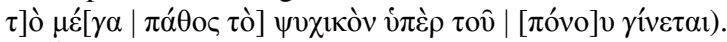

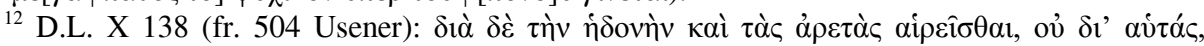

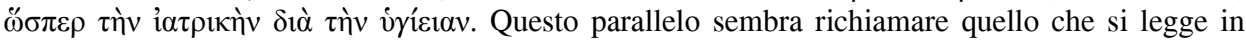
Cicerone (Fin. I 42, fr. 397 Usener), su cui vd. infra.

${ }^{13}$ R.Nat. III 510-512 (cf. anche il fr. 221 Usener: "come la medicina, infatti, non ha nessuna

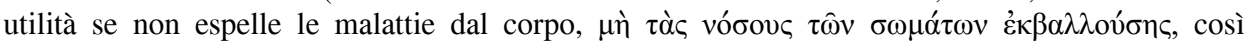
nemmeno la filosofia, se non espelle la passione dall'anima"; su questo punto cf. Voelke, Philosophie, 36). I vv. 486 ss. descrivono i mali più forti dell'anima mostrando i sintomi di una crisi epilettica ad essi correlati. Sul trasferimento nel campo della filosofia morale, soprattutto in Platone e in ambito stoico, della teoria terapeutica ippocratica, cf. Vegetti, Passioni, part. 224 ss.

${ }^{14} \mathrm{Si}$ tratta della versione ampliata di una comunicazione letta al XIV Congresso Internazionale di
} 
tracciava un percorso all'interno della collezione ercolanese, commentando una serie di passi tratti da varie opere, da cui emerge chiaramente il ruolo della filosofia come farmaco dell'anima. Venti anni dopo, Michael Erler, parlando di Philologia medicans ${ }^{15}$, ha messo in luce gli strumenti e i metodi che sono alla base della terapia epicurea nei continuatori della scuola tra II e I sec. a.C.: secondo lo studioso tedesco, è la fedeltà al Maestro, attraverso la lettura continua dei suoi ipsissima verba e la corretta interpretazione dei testi, ad assicurare il saldo possesso della dottrina e il raggiungimento della salute dell'anima.

Intendo ripercorrere alcuni di questi testi, anche alla luce degli studi più recenti, e, nello stesso tempo, proporre una serie di altri frammenti ercolanesi in cui ci sono riferimenti e immagini che richiamano la scienza medica e il mestiere del medico e, laddove il contesto sia più o meno leggibile, da cui sembra

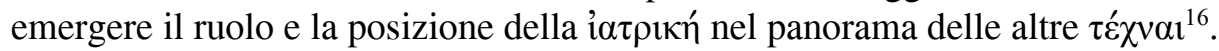

Epicuro, che pose tra i temi fondanti del sistema quelli della sofferenza e della morte, scrisse un'opera Sulle malattie e la morte, come sappiamo da Demetrio Lacone nel P.Herc. 1012, che conserva un'opera priva di titolo sulla filologia dei testi epicurei ${ }^{17}$. Il titolo, secondo Gigante, andrebbe identificato con quello dell'opera di Epicuro che si legge nell'elenco di Diogene Laerzio (X 28) che,

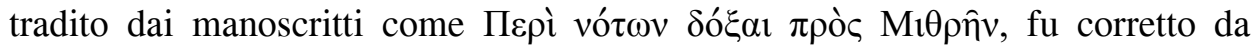

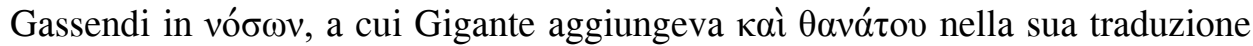
(Opinioni sulle malattie e sulla morte a Mitre $)^{18}$.

Nello stesso papiro 1012 ricorrono interessanti citazioni di testi con cui vengono confrontati i libri del Maestro. Tra questi, compaiono alcune menzioni di testi medici. Amneris Roselli, in un lavoro del 1988, ha ben messo in luce la citazione, da parte di Demetrio Lacone, di un passo del Prorretico I (col. xviii), di Epidemie VI (col. xxi) e di alcune parole di interpretazione, sempre di Epidemie VI, di Apollonio Empirico. I testi ippocratici non erano sconosciuti a

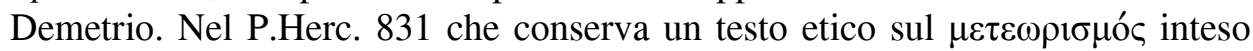
come perturbatio animi in seguito a rappresentazione fallace, anche questo purtroppo privo di titolo, ma attribuito senza dubbio ancora a Demetrio Lacone, nella col. ii Körte si legge una citazione di Prognostico 7 utilizzata da Demetrio forse per istituire un'analogia tra la follia di cui sono preda gli ipocondriaci e quella di chi cade preda di pensieri mostruosi non affidandosi alla sensazione ${ }^{19}$.

Papirologia di Oxford (1974). Sullo stesso tema, in particolare sui notevoli spunti offerti dal Пepì $\pi \alpha \rho \rho \eta \sigma i ́ \alpha \varsigma$ di Filodemo, Gigante è ritornato nel volume Ricerche filodemee (part. pp. 55-113).

${ }^{15}$ Erler, Philologia.

${ }^{16}$ In alcuni casi possediamo ancora il papiro in originale, in altri, laddove il papiro sia stato trattato col metodo della 'scorzatura' che, dopo il disegno, distruggeva gli strati per poter accedere al livello sottostante, è possibile leggere il testo solo negli apografi. Vd. anche infra.

${ }^{17}$ Col. xxvii 3-5 Puglia. Vedi il commento di Puglia, Aporie, 241-2.

${ }^{18}$ Gigante, Vite, 410 e 572. Diversamente, Dorandi, Diogenes, 751, non accetta la proposta di Gigante.

${ }^{19}$ Su questo punto si veda Gigante, Distrazioni, 2. Sui meccanismi e le funzioni di queste e di altre citazioni di Demetrio Lacone e per una bibliografia aggiornata, rimando a Parisi, Citazioni. 
In tutti questi passi demetriaci, Roselli ha riscontrato una certa libertà nel modo di citare il testo ippocratico, con varianti e costrutti che non solo non ricorrono nei manoscritti medievali, ma non trovano riferimento in altri testi dello stesso corpus, dovuta al fatto che il Lacone doveva essere "più attento al senso che non sollecito del rispetto della lettera" e alla circostanza che doveva avere tra le mani del "materiale già abbastanza elaborato" 20 .

L'uso di riportare termini della medicina nell' ambito della trattazione filosofica non era estraneo a Demetrio Lacone ${ }^{21}$. Nel P.Herc. 1055, privo di titolo, che conserva un'opera Sulla forma del dio $^{22}$, come ha sottolineato recentemente Piergiacomi ${ }^{23}$, Demetrio utilizza il termine $\dot{\varepsilon} \pi \imath \pi \alpha \sigma \mu o ́ c$, che si trova già in Ippocrate $^{24} \mathrm{e}$ in Galeno indica un movimento di trazione o aspirazione ${ }^{25}$. In Demetrio, in un contesto del tutto differente, esso designa un tipo di ragionamento inferenziale. Lo stesso uso si riscontra ancora in Filodemo, nell'opera $D e$ signis $^{26}$, in cui, come vedremo, l'inferenza per analogia, sostenuta dall'Epicureo, trova molti riscontri con l'attività diagnostica della medicina ${ }^{27}$. Recentemente

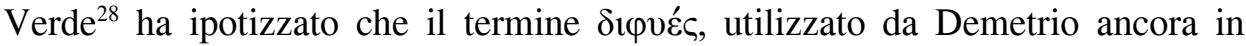
P.Herc. 1055 ([De forma dei]), per affermare che "niente di ciò che è di duplice

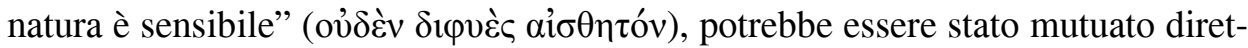
tamente dal campo medico-anatomico ${ }^{29}$.

Tornando a Epicuro, nel P.Herc. 1148, che conserva il XIV libro del Пєрì

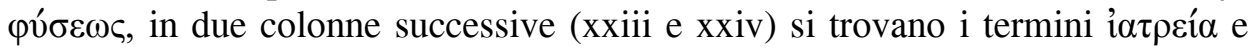

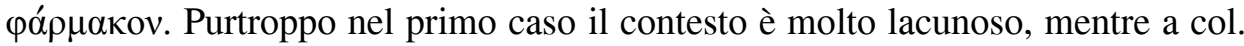
xxiv il testo si lascia seguire più facilmente:

E anche questo è desiderabile, che ognuno invischiato in tali quisquilie abbia, per così dire, un farmaco, per mezzo del quale le rappresentazioni semplicistiche nella contemplazione della natura saranno liberate dal turbamento ad esse congenito, che anche in séguito... ${ }^{30}$

\footnotetext{
${ }^{20}$ Roselli, Citazioni, 55 e 56.

${ }^{21}$ Sulle testimonianze di Erotiano e Fozio dell'interesse di Demetrio Lacone per il testo di Ippocrate, cf. Roselli, Citazioni, 56, Gigante in Puglia, Aporie, 19, 215 ss. e Parisi, Citazioni.

${ }^{22}$ P.Herc. 1055, col. xiv Santoro.

${ }^{23}$ Piergiacomi, Attraction, 12-13.

${ }^{24}$ Cf. Gal. Comm.Epid., CMG V.10.2.2 pp. 300,24-25 e 301,7-302,18 Pfaff.

${ }^{25} \mathrm{Il}$ termine è usato anche più tardi da altri medici come Sorano, Oribasio, Aezio e Paolo di Egina.

${ }^{26}$ P.Herc. 1065, col. xiii 1-8 De Lacy. Su questo papiro, vd. anche infra.

${ }^{27}$ Vd. infra. Così anche in P.Herc. 346, trattato etico epicureo di autore e titolo incerti (col. xii 4-9 Capasso).

${ }^{28}$ Verde, $\triangle I \Phi Y E \Sigma, 167$.

${ }^{29}$ Col. xxii 9-10 Santoro. Cf. Gal. U.P. III 664,16 e 715,1 K.

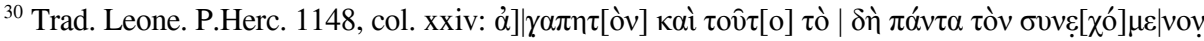


Leone, nell'analisi puntuale dei termini medici e del loro passaggio alla sfera più genuinamente filosofica ${ }^{31}$, sottolinea come "l'assimilazione della terminologia medica da parte di Epicuro si rivela, dunque, profonda e consapevole" 32 .

Lo stesso concetto ritorna chiaramente in un passo dell'epigrafe epicurea di Diogene di Enoanda, che si data al II sec. d.C. Diogene afferma ${ }^{33}$ :

...dal momento che i rimedi dello scritto raggiungono un largo numero di persone, ho voluto usare questo portico per diffondere pubblicamente le medicine che offrono la salvezza, che noi abbiamo completamente sperimentato.

Diogene, con intento quasi programmatico, auspica che i rimedi (il termine $\beta o n ́ \theta \eta \mu \alpha$ è utilizzato anche da Ippocrate con la stessa sfumatura di significato) ${ }^{34}$ possano diffondersi attraverso l'iscrizione collocata sotto il portico e portare la salvezza dell'anima a tutti coloro che, passando di lì, leggano i precetti di Epicuro. Diogene offre i farmaci della filosofia epicurea perché "i più sono in generale contaminati,

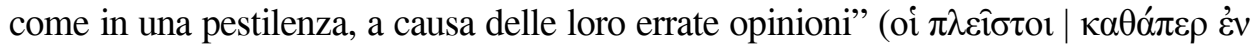

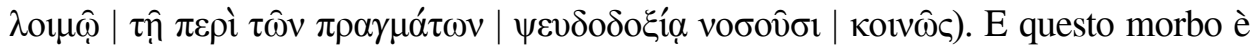
contagioso: "per la reciproca imitazione, infatti, si trasmettono l'un l'altro la malattia come le pecore" (

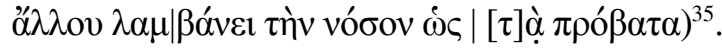

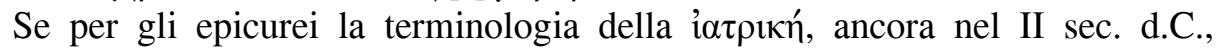
costituisce un reale strumento per spiegare i mali e le conseguenti cure dell'anima (e, come vedremo, essa trovò anche un suo puntuale riconoscimento all'interno del panorama delle $\tau \dot{\varepsilon} \chi v \alpha \iota)$, non è così per la pratica della confezione e della vendita dei farmaci. Seguendo un'idea molto diffusa nell'antichità, in un

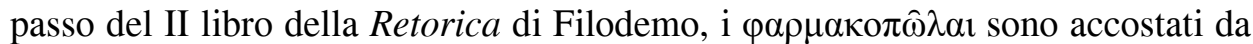
un avversario agli indovini e a coloro che, per i loro falsi ragionamenti, sono

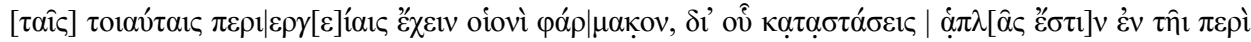

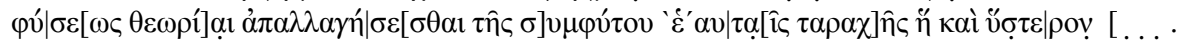

${ }^{31}$ Leone, Epicuro, 76.

${ }^{32}$ La studiosa (p. 77) richiama anche un passo di Temistio (Paraphr.Aristot.Phys. Z 1 = fr. 278 Usener) in cui il riferimento epicureo al "farmaco dell'anima" è menzionato con toni sarcastici:

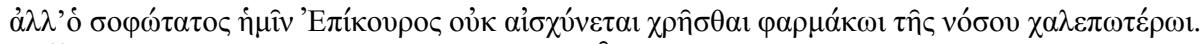

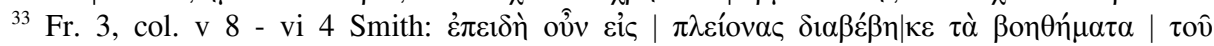

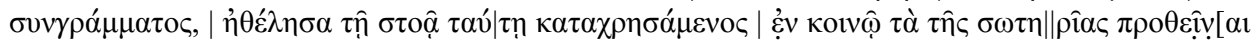

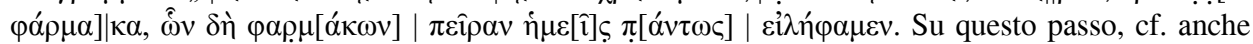
Hammerstaedt, Strategie, 140-1, che parla di "messaggio salutare".

${ }^{34}$ Virg.Morb. 13.

${ }^{35}$ Fr. 3, col. iv 13 Smith. La trad. è di Casanova, rivista da Hammerstaedt, Strategie, 140. Come ha acutamente sottolineato Smith, Diogenes, 439, il paragone con il contagio della peste nelle pecore, ben presente in Tucidide (II 51,4: ڤँ $\sigma \pi \varepsilon \rho \tau \grave{\alpha} \pi \rho o ́ \beta \alpha \tau \alpha$ ) e ripreso da Lucrezio (VI 1237: lanigeras tamquam pecudes et bucera saecla), è "particularly appropriate because of the animals' proverbial stupidity and their habit of following one another". 
assimilabili ai sofisti ${ }^{36}$.

Il parallelo tra medicina e retorica in Filodemo si trova spesso all'interno della trattazione del problema della individuazione della natura dell'arte retorica e, di conseguenza, della distinzione delle arti in due grandi categorie, di ascendenza

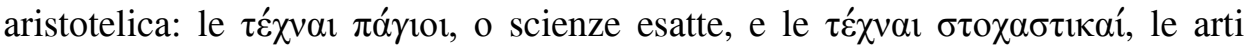
congetturali, basate sull'inferenza dai segni, e quindi su una previsione congetturale basata sul verosimile ${ }^{37}$. Sia la medicina sia la retorica appartengono a questa seconda categoria.

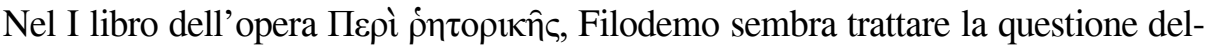
l'oggetto della retorica servendosi, in parallelo, del discorso di Socrate nel Gorgia platonico $^{38}$. L'Epicureo riprende da Platone gli esempi di $\tau \dot{\varepsilon} \chi v \alpha \alpha$, di cui, a differenza di quanto accade con la retorica, si possa individuare senza difficoltà l'oggetto.

Sia nel dialogo sia nel passo di Filodemo, tra queste $\dot{\varepsilon} \pi \imath \tau \hat{\jmath} \mu \alpha$, oltre alla tessitura, alla musica e alla ginnastica, figura anche la medicina. È stato merito di Federica Nicolardi proporre una diversa interpretazione del passo sul fondamento di una nuova sistemazione del testo dell'apografo napoletano ${ }^{39}$. Secondo questa ricostruzione, Filodemo, a partire dalla domanda posta da

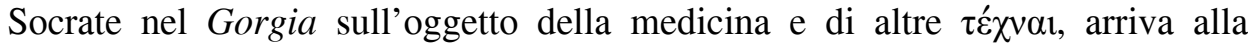
definizione dell'oggetto della retorica. Se tale ricostruzione è corretta, Filodemo

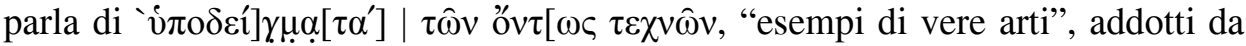
Platone (1l. 7-8). A causa della frammentarietà del passo del I libro del $D e$ rhetorica, non è possibile stabilire con certezza se Filodemo si riconoscesse in questa descrizione delle "vere arti" e nell'idea, espressa nel dialogo da Socrate,

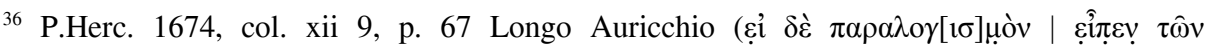

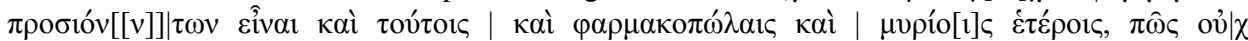

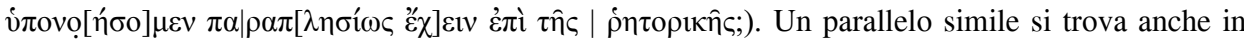

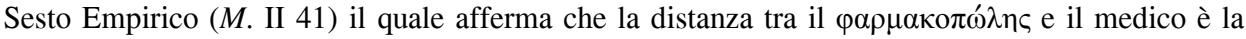
stessa che passa tra il demagogo e il politico (cf. in proposito Fausti, Farmacologia, 24).

${ }^{37}$ Cf. il II libro De rhetorica (P.Herc. 1674), coll. xxxviii 35 - xxxix 24 Longo Auricchio. In particolare, come si evince da un passo dello stesso II libro (col. xxxiv 11-31 Longo Auricchio), Bromio, un altro epicureo, amico di Filodemo, si era proposto di scrivere sulla classificazione delle discipline, verificando quali (tra queste la stessa medicina), potessero essere annoverate tra le arti

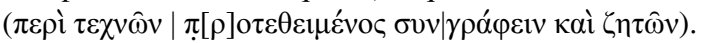

${ }^{38}$ In particolare Plat. Gorg. 449d-450e.

${ }^{39}$ Nicolardi, Elementi, 60 ss. Il frammento disegnato, trovandosi all'esterno del rotolo originario, era stato rimosso e gli strati successivi disegnati e distrutti per accedere via via a quelli sottostanti, con la tecnica cosiddetta della scorzatura. La studiosa, ripristinando la lettura del passo del Gorgia nel margine inferiore del disegno napoletano 4 del P.Herc. 250, è riuscita a riconnettere questo disegno con il disegno n. 6 del P.Herc. 1612 che costituiva, prima della scorzatura, la parte superiore della stessa colonna. La definizione platonica della medicina, che "rende capace di pensare agli ammalati e

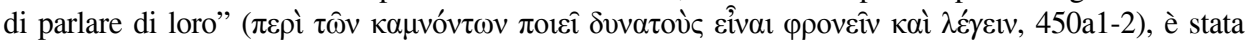
citata da Filodemo, ma, omessa dallo scriba, è stata poi ripristinata nel margine inferiore. Nel punto in cui il passo deve essere ricollocato, nella porzione superiore della colonna, troviamo nel disegno l'avverbio кát $\omega$ che rimandava all'inserimento nel margine. 
che la definizione di retorica come arte dei discorsi non sia sufficiente.

Un altro riferimento alla medicina in parallelo con il Gorgia si trova ancora nel I libro del De rhetorica ${ }^{40}$. Nonostante la parte superiore della colonna non sia conservata, sembra che qui Filodemo stia discutendo le diverse interpretazioni del concetto di arte, di cui l'autore presenta varie possibili definizioni: all'idea di $\tau \varepsilon_{\varepsilon} \chi \vee \eta$ come attività basata su particolari principi teorici e procedimenti metodici (come la grammatica) è contrapposta quella secondo cui solo la бopía è arte. A questo proposito, Filodemo ricorda la concezione platonica della $\tau \dot{\varepsilon} \chi \vee \eta$, che deve avere in sé il principio razionale attraverso cui possa comprendere e giustificare

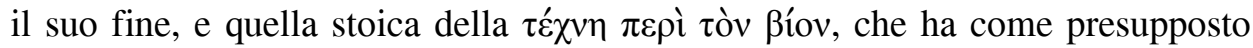

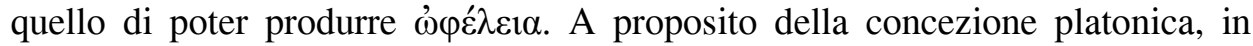
Filodemo leggiamo che "altri [considerano arte] quella che tiene conto (della natura) 'di ciò che somministra a colui a cui lo somministra', come dice Platone" ${ }^{41}$. La citazione proviene da un passo del Gorgia molto dibattuto ${ }^{42}$, in cui Socrate spiega a Polo che non considera la ỏ оолоикń, l'arte della cucina, una $\tau \dot{\varepsilon} \chi \vee \eta$, ma solo una semplice $\dot{\varepsilon} \mu \pi \varepsilon i p i ́ \alpha$, poiché essa, a differenza della medicina, non somministra un preciso alimento sulla base di criteri razionali. Dal contesto di queste linee e dalle colonne successive, non sembra che Filodemo si trovi d'accordo con nessuna delle definizioni di $\tau \dot{\varepsilon} \chi v \eta$ citate.

Se, quando si serve del Gorgia platonico nel I libro del De rhetorica, Filodemo sembra mettere la retorica e la medicina in opposizione, o comunque non sembra creare un parallelo tra esse, in altri luoghi della sua opera retorica tra le due arti è attestato talvolta un rapporto di alterità, talaltra di similaritầ ${ }^{43}$. Nel P.Herc. 1004 (libro VII del De rhetorica di Filodemo" ${ }^{44}$ ), si legge che "il discorso secondo il quale gli stili di vita proposti da noi sono salutari convince chi vuole vivere in salute più di quella ( $s c$. la medicina)": nel passo si sottolinea, dunque, la maggiore persuasività del retore rispetto al medico nell'approccio al malato; tuttavia, questa utilità del discorso è solo superficiale, poiché essa, lontana dall'apportare reali benefici al malato, ha il solo scopo di far trarre profitto. È basato, invece, sull'analogia l'accostamento tra le due parti presentato in P.Herc. $1004^{45}$, ottimamente riedito in un recente articolo da Matilde Fiorillo, in cui

\footnotetext{
${ }^{40}$ Questa volta nella parte finale del papiro (P.Herc. 1427) il cosiddetto 'midollo' aperto con la celebre macchina di Piaggio.

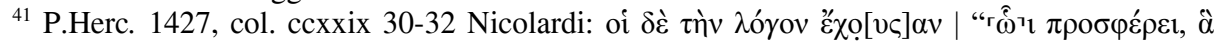

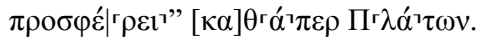

$42465 \mathrm{a} 2$ ss. Longo Auricchio richiama anche Plat. Gorg. 503e, il cui contesto è affine al passo citato letteralmente da Filodemo; un'espressione simile ritorna anche a 501a. Sulle divergenze tra i manoscritti che trasmettono il dialogo (in particolare, a proposito dell'aggiunta della congiunzione $\eta \eta$ tra le due relative da parte di Dodds) e sulla ripresa filodemea, rimando al commento all'edizione del primo libro del De rhetorica di F. Nicolardi (Nicolardi, Filodemo, 46-8).

${ }^{43}$ Fiorillo, Medico, 202 ss.

${ }^{44}$ Col. iii 4-18 Sudhaus I, p. 284.

${ }^{45}$ Col. xlvi 1-19 Sudhaus I, pp. 345-6. Nei passi tratti da questo papiro non è sempre chiaro se
} 
Filodemo affronta il problema della fallibilità delle arti congetturali e della

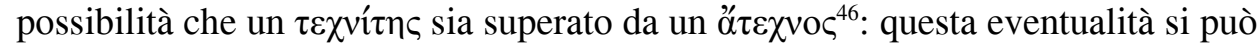
verificare sia per un medico che per un retore (ma per quest'ultimo soltanto nell'ambito concreto dei $\pi \rho \alpha ́ \gamma \mu \alpha \tau \alpha$ e non in quello esclusivamente tecnico dell'eloquenza), "poiché talora sono superati da profani della disciplina ( $\dot{v} \pi^{\prime}$ i $\delta 1 \omega \tau \hat{\omega} v)$ che hanno scoperto una qualche medicina capace di curare una malattia, mentre loro la ignorano". Nella colonna $1 x^{x x i x}{ }^{47}$ si può ricostruire il parallelo tra il medico che non può guarire i malati collettivamente ( $\dot{\alpha} \theta \rho o ́ o v \varsigma)$, ma uno per uno allo stesso modo in cui il retore deve ingraziarsi gli ascoltatori singolarmente $\left.(\kappa] \alpha \theta^{\prime} \check{\varepsilon} v \alpha\right)$. In un altro passo del settimo libro della Retorica ${ }^{48}$, vengono menzionate ancora la medicina e la retorica, questa volta insieme all'arte nautica, come già si affermava nello pseudoplatonico Epinomide ${ }^{49}$. Esse

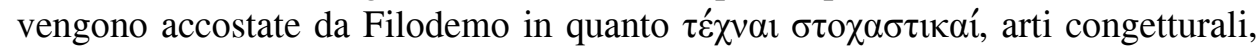
che, come ho già detto, procedono attraverso argomentazioni o indizi: come

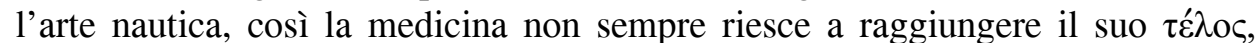
basandosi sull' eíkóc, cioè sulla valutazione del "probabile". Anche Cicerone, nel De finibus, afferma che così come l'arte del medico e quella del timoniere sono lodate non per se stesse, ma per l'utilità che producono, allo stesso modo la sapienza è ricercata come artefice del suo fine e dell'ottenimento del piacere ${ }^{50}$. Un parallelo molto simile si trova nel II libro del De rhetorica: nell'elencazione di alcune attività che non promettono di compiere sempre il fine che si sono prefisse

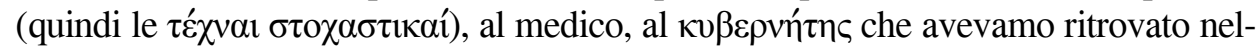

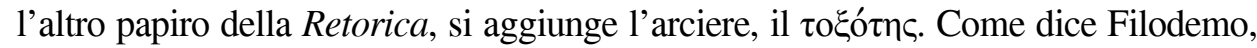
si tratta di "tutti quelli che non hanno delle scienze stabili, ma congetturali"

sia rappresentato il pensiero di Filodemo o quello dei suoi avversari.

${ }^{46}$ Il concetto torna anche in P.Herc. 1427 (De rhetorica I), fr. 3,13-23 e in P.Herc. 1674 (De

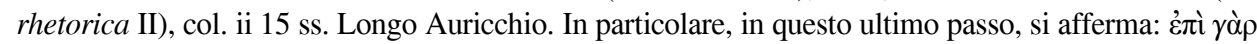

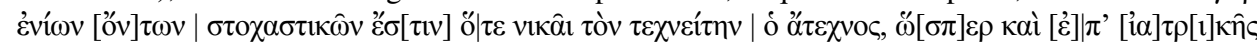

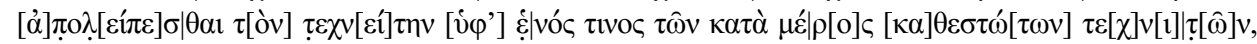

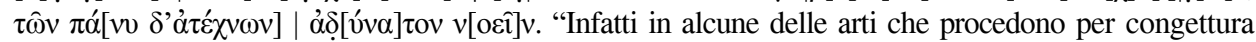
càpita talvolta che il non artista superi l'artista, come anche avviene nella medicina che il tecnico sia superato da uno di quei tecnici che hanno competenza parziale, ma di coloro che siano del tutto inesperti è impossibile concepirlo" (trad. Longo Auricchio, p. 47).

${ }^{47}$ Sudhaus I, pp. 370-1. Cf. anche il III libro De rhetorica (P.Herc. 1506, col. lv 21-28= P.Herc. 1426, coll. x a 25-xi a 2 e xiii a 24-30 Hammerstaedt).

${ }^{48}$ Trattato anche questo da Fiorillo, Medico, 205-6 (P.Herc. 1004, col. xciii 1-9 Sudhaus I, p. 373).

49 976a-b.

${ }^{50}$ I 42 (fr. 397 Usener): ut enim medicorum scientiam non ipsius artis, sed bonae valetudinis causa probamus, et gubernatoris ars, quia bene navigandi rationem habet, utilitate, non arte laudatur, sic sapientia, quae ars vivendi putanda est, non expeteretur, si nihil efficeret; nunc expetitur, quod est tamquam artifex conquirendae et comparandae voluptatis. Per lo stesso parallelo si veda ancora Filodemo, P.Herc. 468 (Philod. Rhet. III), fr. VI Hammerstaedt e Platone, Resp. I 341c-342e.

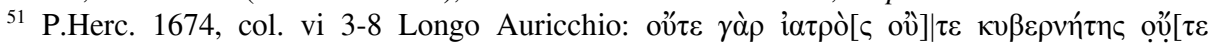


E proprio a questo proposito, nel P.Herc. 1079, che è una scorza, cioè una porzione esterna del II libro del De rhetorica, Filodemo afferma che anche se il medico avrà salvato centinaia di malati di elefantiasi o decine di sofferenti ai

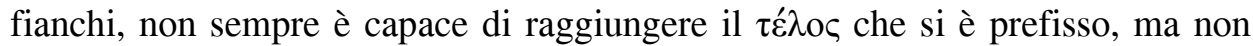
per questo non potrà essere definito un $\tau \varepsilon \chi v^{\prime}$ ín c adeguato ed esperto $^{52}$.

Sul versante più spiccatamente lessicale l'opera ercolanese in cui il linguaggio filosofico riprende più da vicino il lessico medico è il trattato Sulla libertà di parola di Filodemo, che, è importante ricordarlo, come è dichiarato nel titolo, si presenta come una trattazione delle lezioni di Zenone Sidonio ${ }^{53}$, maestro di Filodemo, che fu caposcuola epicureo ad Atene nel I sec. a.C. e di cui anche Cicerone seguì le lezioni ${ }^{54}$. Si può facilmente intuire il motivo della scelta di un linguaggio che, in molti casi, si sovrappone a quello medico: il filosofo è un terapeuta che ha il compito di guidare e curare l'anima dell'allievo grazie alla $\pi \alpha \rho p \eta \sigma i ́ \alpha$, la franchezza nel parlare. Ed è proprio la parola lo strumento attraverso cui si attua tale funzione curativa. Nel fr. 84 si parla di coloro che esercitano in maniera esitante la franchezza davanti agli amici e di quando, in tale contesto,

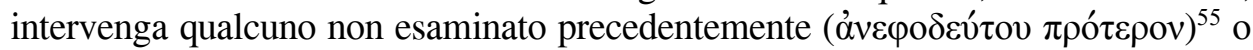

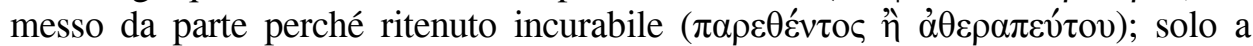
questo punto, avvenuta la prognosi, è possibile l'intervento terapeutico ${ }^{56}$. La scelta del termine á $\theta \varepsilon \rho \alpha ́ \pi \varepsilon v \tau o \varsigma$ si inscrive chiaramente nell'attività del filosofo-medico che ha il compito di guidare e guarire il discente, a patto che egli non sia

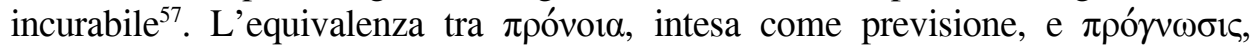
messa in luce per questo passo da Gigante ${ }^{58}$, è un caposaldo della medicina

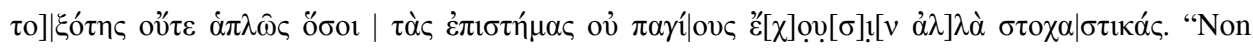
(promette di compiere il fine) il medico né il timoniere né l'arciere né, per farla breve, tutti quelli che non hanno delle scienze stabili, ma congetturali" (trad. F. Longo Auricchio).

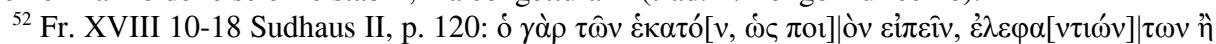

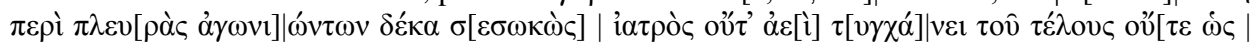

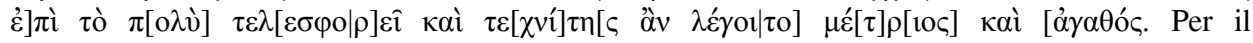
supplemento $\tau \varepsilon \lambda[\varepsilon \sigma \varphi \circ \mid \rho] \varepsilon \hat{\imath}$ (Sudhaus scriveva $\tau \varepsilon \lambda[\varepsilon \sigma 10 v \rho \mid \gamma] \varepsilon \hat{i})$ cf. Gigante, Philosophia, 55 n. 55. Contro l'elefantiasi, molto diffusa tra I sec. a.C. e I sec. d.C. e che per Galeno è causata da un eccessivo accumulo di bile nera, era comunemente utilizzata la carne di vipera, molto rinomata

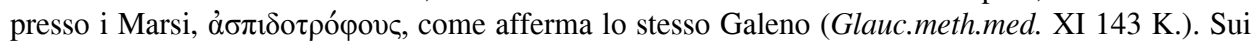
dolori ai fianchi citati nel De ira vedi infra.

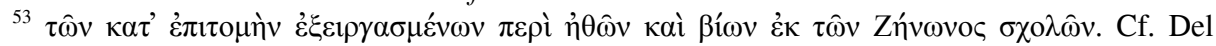
Mastro, Titoli, 314-7.

${ }^{54}$ Cic. Fin. I 16; Nat.deor. I 59; Acad. I 46; Tusc. III 38 e Nat.deor. I 93-94 = Zeno, frr. 5-9 Angeli/Colaizzo. Su Zenone Sidonio mi limito a rimandare alla raccolta curata da Angeli/Colaizzo, Frammenti e a Del Mastro, Lode.

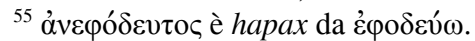

${ }^{56} \mathrm{Cf}$. Gigante, Philosophia, 57.

${ }^{57}$ Cf. Gigante, Philosophia, 56 e Ricerche, 72, che rileva un parallelismo tra gli $\dot{\alpha} \theta \varepsilon \rho \alpha ́ \pi \varepsilon v \tau o$ del $D e$

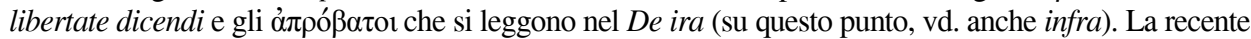
edizione americana (Konstan/Clay/Glad/Thom/Were, Philodemus, 88-9) traduce "untreatable".

${ }^{58}$ Gigante, Philosophia, 57. 
ippocratica, come si legge fin dal primo capitolo del Prognostico ${ }^{59}$.

A proposito del paragone tra i mali dell'anima e quelli del corpo, anche nel Пєрі̀ ò $ү \hat{\eta ি ৎ ~(P . H e r c . ~ 182) ~ F i l o d e m o ~ r i c o r d a ~ c h e ~ g l i ~ a d i r a t i ~ n e c e s s a r i a m e n t e ~ n o n ~}$ riescono a progredire, perché "non sopportano né maestri né compagni di scuola, qualora li rimproverino e li correggano, come le ulcere maligne non sopportano le somministrazioni dei farmaci lenitivi" ${ }^{\prime 60}$. E in un altro luogo della stessa opera, gli adirati sono assimilati agli epilettici ${ }^{61}$. In questo punto del testo ${ }^{62}$, gli iracondi sono descritti come malati della cui malattia vengono dettagliatamente esposti i sintomi e le conseguenze fisiche:

Il fatto che a molti l'ira generò sia questo (cioè uno stato depressivo) sia ciò che ne deriva, vale a dire rotture di polmoni e dolori ai fianchi e molte altre affezioni apportatrici di morti, è possibile sentire dai medici e sono in grado di osservarlo quelli che esaminano attentamente costoro.

Più avanti, nella descrizione dei sintomi dell'ira, si afferma che mentre coloro che assistono gli iracondi non devono fare nulla che possa perturbarli, i medici possono indurre in loro un'eccitazione (evidentemente a scopo diagnostico o terapeutico, ma purtroppo qui il testo si interrompe $)^{63}$. Come possiamo osservare, $\mathrm{i}$ modi della trattazione filosofica, che ricalcano i procedimenti della medicina (diagnosi-terapia-prognosi), sono molto simili nelle opere Sull'ira e Sulla libertà di parola: in entrambi i casi viene individuata una patologia che deve essere innanzitutto analizzata e, successivamente, grazie ai $\varphi \alpha ́ \rho \mu \alpha \kappa \alpha$ che l'Epicureismo mette a disposizione dell'uomo, essa può essere curata e debellata ${ }^{64}$.

Il parallelo con la medicina diventa più stringente nei frr. 63-64 del Пєрì $\pi \alpha \rho \rho \eta \sigma i ́ \alpha c:$ nel fr. 64 si afferma che il filosofo

non avendo ottenuto alcun risultato, praticherà ancora [la franchezza] verso la

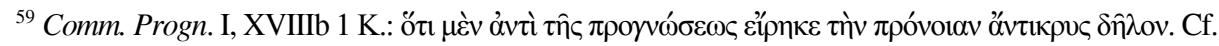
anche ibid., XVIIIb 4 K. Su questo punto rimando all'interessante lavoro di Fausti, Segno, part. 262-3.

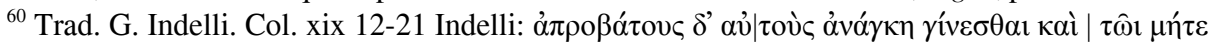

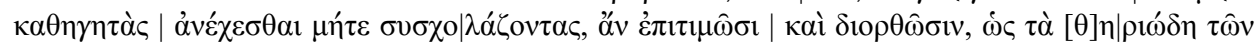

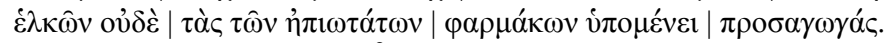

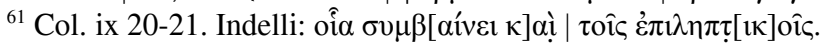

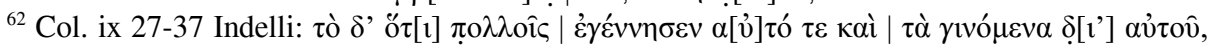

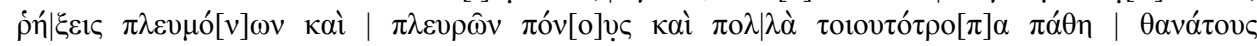

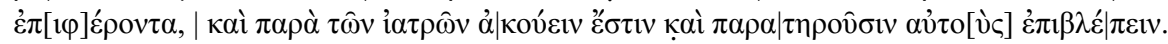

${ }^{63}$ Col. x 40 Indelli.

${ }^{64}$ Più volte Gigante è tornato sulle affinità tra le due opere che facevano parte del complesso

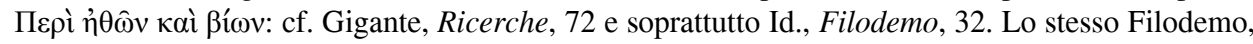
inoltre, nel De ira (col. xxxvi 24 s. Indelli) fa riferimento proprio all'opera Sulla libertà di parola in cui si trattava anche dei sapienti che sono predisposti all'ira. 
stessa persona. Se, avendo sbagliato, non reagisce alla franchezza, il maestro parlerà con franchezza ancora una volta. Infatti, se un medico, per quanto riguarda la stessa malattia, non ha ottenuto alcun risultato con un clistere, purgherà ancora una volta... ${ }^{65}$

E nel frammento precedente (fr. 63):

È quasi come quando un medico suppone attraverso segni ragionevoli che qualcuno abbia bisogno di una purga $(\kappa \varepsilon ́ v \omega \mu \alpha)$, e poi, avendo sbagliato nella diagnosi, non purga mai più costui se è afflitto da un'altra malattia. Così, riflettendo attraverso questa stessa cosa, egli parlerà ancora con franchezza ${ }^{66}$.

In questo punto, non è sfuggito a Gigante il confronto con la filosofia scettica, di

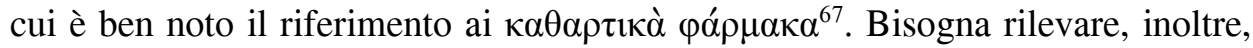
l'uso dei termini $\sigma \eta \mu \varepsilon \hat{\imath} 0 v-\sigma \eta \mu \varepsilon i ́ \omega \sigma \iota^{68}$ : per gli Epicurei, dal momento che i piani medico-terapeutico e filosofico vengono a sovrapporsi, si può affermare che la semiotica, intesa come scienza dei segni nel loro complesso, viene riportata alla semeiotica, intesa come scienza dell'interpretazione dei segni in quanto sintomi. L'azione del medico che pratica la dissezione applicando il bisturi ai malati è assimilata a quella dell'educatore che deve spronare la correzione degli errori ${ }^{69}$.

$\mathrm{Ma}$, ancora nel De libertate dicendi, il parallelo è trattato anche a un livello più pratico, attraverso la menzione dell'elleboro ${ }^{70}$ che, come sappiamo, era cita-

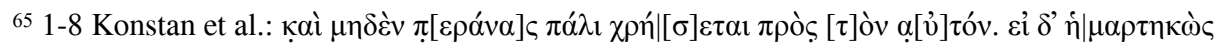

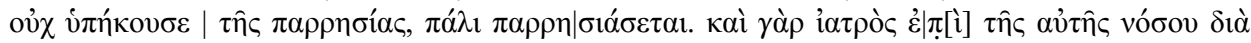

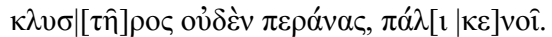

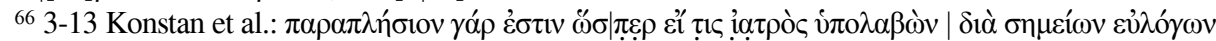

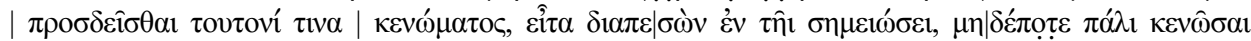

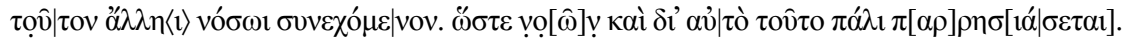

${ }^{67}$ Gigante, Philosophia, 58 e n. 111. Lo studioso riteneva che "tale punto di contatto possa contribuire a chiarire il carattere contaminato del tardo epicureismo". Nel De signis, Filodemo risponde alla settima delle obiezioni mosse alle tesi del compagno di dottrina Bromio, che riportava le teorie di Zenone Sidonio (col. xx 11-21 De Lacy): gli avversari rimproverano che l'inferenza secondo similarità non è sempre possibile. Ad esempio le carni bovine non sono ugualmente digeribili per ogni essere umano. Da qui deriva che le affermazioni degli Epicurei sul concetto di similarità non sono affidabili. Filodemo risponde (P.Herc. 1065, col. xxv 38-39 De

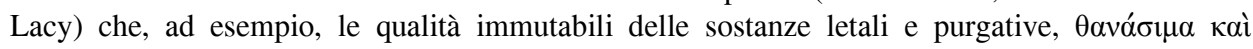
$\kappa \alpha \theta \alpha \rho \tau \iota \kappa \alpha ́$, costituiscono una prova a favore della inferenza secondo similarità.

${ }^{68}$ Sulla semeiotica come osservazione dei segni nella medicina ippocratica (e sulle relative implicazioni sul piano più squisitamente lessicale), rimando a Fausti, Segno, part. 260 ss.

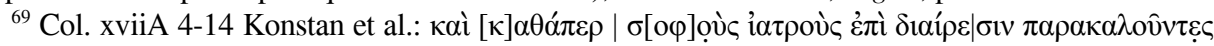

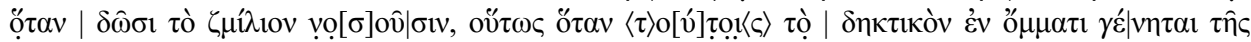

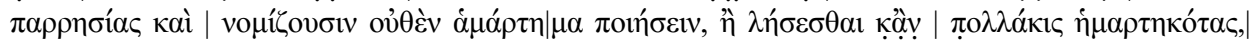
$\pi \alpha \rho \alpha \kappa \alpha \lambda o v \sigma \sigma \mathrm{vo}[v] \theta \varepsilon \tau \varepsilon i v$. Su questo passo cf. anche Voelke, Philosophie, 48.

${ }^{70}$ Sugli usi nella medicina ippocratica delle diverse tipologie di ellèboro, cf. Aliotta/Piomelli/ 
to, già a partire da Ippocrate, come purgante: il passo è particolarmente lacunoso, ma si fa riferimento a coloro che, bevendo l'estratto di questa pianta, non dovevano apparire ridicoli ai medici che ben conoscevano i suoi effetti nefasti ${ }^{71}$.

Tra le piante medicinali, anche l'assenzio ${ }^{72}$ è ricordato da Filodemo, nella stessa opera De libertate dicendi e ancora nel De ira. In particolare, in quest'ultimo caso, è presente la bellissima immagine del sapiente che, irato, è costretto a praticare una punizione malvolentieri come se stesse bevendo

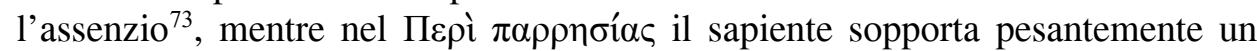
atteggiamento di biasimo come se bevesse l'assenzio ${ }^{74}$. Non possiamo non ricordare in proposito, $i$ versi lucreziani in cui il poeta dichiarava di trattare la difficile dottrina epicurea come coloro che curano i fanciulli, passando sugli orli dei bicchieri pieni di assenzio "il dolce liquido biondo del miele"75.

\section{Bibliografia}

Aliotta/Piomelli/Pollio/Touwaide, Piante $=$ Giovani Aliotta $/$ Daniele Piomelli $/$ Antonino Pollio $/$ Alain Touwaide, Le piante medicinali del "Corpus Hippocraticum" (Hippocratica Civitas 5), Milano 2003.

Angeli, Amici = Anna Angeli (ed.), Filodemo, Agli amici di scuola (La Scuola di Epicuro 7), Napoli 1988.

Angeli, Compendi = Anna Angeli, Compendi, Eklogai, Tetrapharmakos: due capitoli di dissenso nell'Epicureismo, CErc 16 (1986), 53-66.

Angeli/Colaizzo, Frammenti $=$ Anna Angeli $/$ Maria Colaizzo, I frammenti di Zenone Sidonio, CErc 9 (1979), 47-133.

De Lacy, Methods = Phillipp H. De Lacy / Estelle A. De Lacy (edd.), Philodemus. On Methods of Inference (La Scuola di Epicuro 1), Napoli 1978.

Pollio/Touwaide, Piante, 126-8.

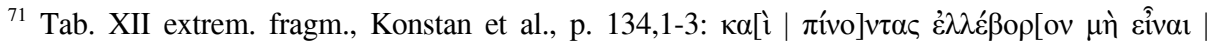

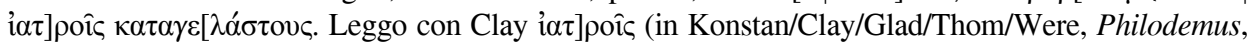

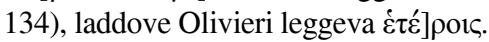

${ }^{72}$ Sugli usi nella medicina ippocratica rimando ad Aliotta/Piomelli/Pollio/Touwaide, Piante, 64-5.

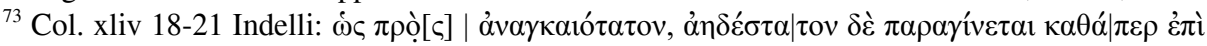

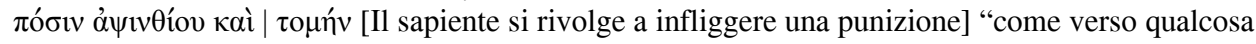
di assolutamente necessario, che, tuttavia, risulta sgradevolissimo, come quando si beve assenzio o si è sottoposti ad un taglio" (trad. G. Indelli). Su questo passo cf. Gigante, Ricerche, 70.

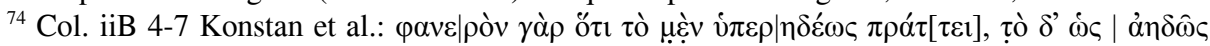

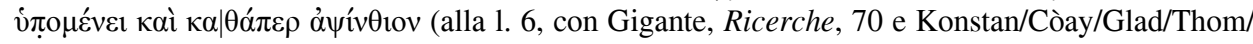

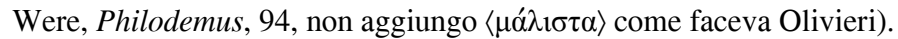

${ }^{75}$ I 936-938: sed veluti pueris absinthia taetra medentes / cum dare conatur, prius oras pocula circum / contingunt mellis dulci flavoque liquore. Per questo passo lucreziano, cf. Gigante, Ricerche, 71 e n. 81 (cf. anche Id., Philosophia, 58), oltre a indicare la stessa immagine in Ps.Plut. Lib.ed. 13d e a ricordare, sulla scia di Ernout, anche Plat. Leg. 659e, non esclude che "Lucrezio forse conosceva questi passi filodemei quando trasponeva nell'amaro infuso dell'assenzio la dottrina epicurea". 
Del Mastro, Lode = Gianluca Del Mastro, Filodemo e la Lode di Zenone Sidonio: $\Pi I \Sigma T O \Sigma$

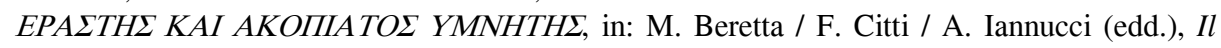
culto di Epicuro. Testi, iconografia e paesaggio, Firenze 2014, 89-109.

Del Mastro, Titoli $=$ Gianluca Del Mastro, Titoli e annotazioni bibliologiche nei papiri greci di Ercolano (CErc 44 Suppl. 5), Napoli 2014.

Dorandi, Diogenes = Tiziano Dorandi (ed.), Diogenes Laertius, Lives of Eminent Philosophers, Cambridge 2013.

Erler, Philologia $=$ Michael Erler, Philologia medicans. Wie die Epikureer die Texte ihres Meisters lasen, in: W. Kullmann / J. Althoff (edd.), Vermittlung und Tradierung von Wissen in der griechischen Kultur (ScriptOralia 61), Tübingen 1993, 281-303.

Fausti, Farmacologia = Daniela Fausti, La farmacologia antica. Un ponte fra irrazionale e razionale, in: V. Boudon-Millot / S. Buzzi (edd.), Guérison, religion et raison. De la médicine Hippocratique aux neurosciences, Paris 2017, 17-39.

Fausti, Segno = Daniela Fausti, Il segno e la prognosi nel Corpus Hippocraticum (Prognostico e Prorretico I e II), "I Quaderni del Ramo d'Oro on-line" 1 (2008), 258-78, URL: <http://www. qro.unisi.it/frontend/node/19>.

Fiorillo, Medico = Matilde Fiorillo, Il medico, il timoniere e il retore in Filodemo, Retorica VII (PHerc. 1004), CErc 42 (2012), 193-208.

Gigante, Distrazioni = Marcello Gigante, Distrazioni su Demetrio Lacone, in: M. Capasso / G. Messeri Savorelli / R. Pintaudi (edd.), Miscellanea Papyrologica in occasione del bicentenario dell'edizione della Charta Borgiana, Firenze 1990, 1-2.

Gigante, Filodemo = Marcello Gigante, Filodemo in Italia, Firenze 1990.

Gigante, Philosophia = Marcello Gigante, Philosophia medicans in Filodemo, CErc 5 (1975), 53-61.

Gigante, Ricerche $=$ Marcello Gigante, Ricerche filodemee, Napoli $1983^{2}$.

Gigante, Vite = Marcello Gigante (ed.), Diogene Laerzio, Vite dei filosofi, Roma/Bari $1998^{3}$.

Jaeger, Paideia III = Werner Jaeger, Paideia. Die Formung des griechischen Menschen, III, Berlin/Leipzig 1947 [trad. it. a cura di A. Setti, Firenze 1959, 1978, 1999].

Hadot, Philosophie = Pierre Hadot, Qu'est-ce que la philosophie antique, Paris 1995.

Hammerstaedt, Strategie = Jürgen Hammerstaedt, Strategie di persuasione all'Epicureismo nell'iscrizione di Diogene di Enoanda, in: M. Beretta / F. Citti / A. Iannucci (edd.), Il culto di Epicuro. Testi, iconografia e paesaggio, Firenze 2014, 139-50.

Hessler, Epikur = Jan Erik Hessler, Epikur Brief an Menoikeus. Edition, Übersetzung, Einleitung und Kommentar, Basel 2014.

Indelli, Ira = Giovanni Indelli, Filodemo, L'ira (La Scuola di Epicuro 5), Napoli 1988.

Konstan/Clay/Glad/Thom/Were, Philodemus = David Konstan / Diskin Clay / Clarence E. Glad / Johan C. Thom / James Were (edd.), Philodemus. On Frank Criticism, Atlanta 1998.

Leone, Epicuro = Giuliana Leone, Epicuro, Della natura, Libro XIV, CErc 14 (1984), 17-107.

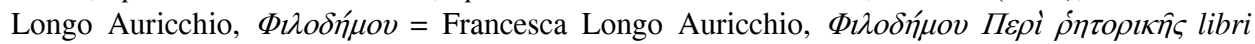
primus et secundus, in: Ricerche sui Papiri Ercolanesi, III, Napoli 1977.

Nicolardi, Elementi = Federica Nicolardi, Elementi per la ricostruzione del I libro del De rhetorica di Filodemo, CErc 45 (2015), 55-65.

Nicolardi, Filodemo $=$ Federica Nicolardi, Filodemo, Il primo libro della Retorica, La Scuola di Epicuro, Napoli 2018.

Nussbaum, Therapy = Martha C. Nussbaum, The Therapy of Desire. theory and Practice in Hellenistic Ethics, Princeton 1994.

Olivieri, ПАРРНСТА $\Sigma=$ Alessandro Olivieri, Philodemi ПЕРI ПАРРНГIA libellus, Lipsiae 1914.

Parisi, Citazioni = Antonio Parisi, Citazioni e meccanismi di citazione nei papiri di Demetrio Lacone, in: Proceedings of the 26th International Congress of Papyrology, Barcelona 2016, in corso di stampa. 
Piergiacomi, Attraction = Enrico Piergiacomi, Mental Attraction to a Magnet-Like God. Demetrius

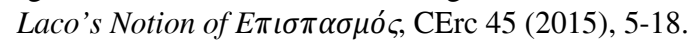

Puglia, Aporie = Enzo Puglia, Demetrio Lacone, Aporie testuali ed esegetiche in Epicuro (La Scuola di Epicuro 8), Napoli 1988.

Roselli, Citazioni = Amneris Roselli, Citazioni ippocratiche in Demetrio Lacone (PHerc. 1012), CErc 18 (1988), 53-57.

Schmid, Epikur = Wolfgang Schmid, Epikur, in: Reallexikon für Antike Christentum, V, 1961, 681-819 [trad. it. a cura di I. Ronca, Epicuro e l'epicureismo cristiano, Brescia 1984].

Vegetti, Passioni = Mario Vegetti, Tra passioni e malattia . Pathos nel pensiero medico antico, "Elenchos" 16 (1995), 219-30.

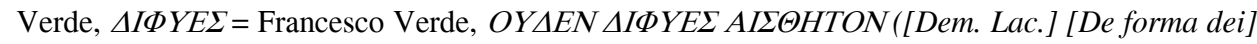
PHerc. 1055, col. XXII 9-10 Santoro, "Vichiana" 54 (2017), 163-7.

Voelke, Philosophie $=$ André-Jean Voelke, La philosophie comme thérapie de l'âme, Etudes de philosophie hellenistique, Fribourg $1993^{2}$. 